\title{
Design and Development of Control System for Three- Dimensional Wireless Human-Computer
}

\author{
Fan $\mathrm{Wu}^{1}$, Mingxin Yuan ${ }^{1,2, *}\left({ }^{*}\right)$, Zhanyu Meng ${ }^{1}$, Dunzhi Chen ${ }^{1}$ \\ ${ }^{1}$ (Zhangjiagang Campus, Jiangsu University of Science and Technology, Zhangjiagang, China) \\ ${ }^{2}$ (Suzhou Institute of Technology, Jiangsu University of Science and Technology, Zhangjiagang, China)
}

\begin{abstract}
In order to improve the convenience and efficiency of the human-computer interaction, a new threedimensional wireless and interactive control system is designed in this paper. The microcontroller MSP430 is taken as the core of the control system, and the gyroscope and acceleration module based on MPU6050 chip is used to detect the state of the gestures. The integrated chip HT82M92A is used to realize the function of mouse driver, and the wireless module based on NRF24L01+ chip is used to realize the wireless communication. Experimental results show that the controller can collect the rotating and tilting gestures and realize the control of the moving cursor. In addition, the controller has many key response functions of left, right, page up and page down keys during the interaction, and has the deictic function by a laser. This control system meets the needs of the human-computer interaction by merging the functions of three-dimensional wireless mouse and flip laser pointer.
\end{abstract}

Keywords: Control system; Human-computer interaction; three dimension; Gestures state

\section{Introduction}

In human-computer interaction, the flip laser pointer has been the protagonist of applications, and its functions develop from simple multimedia page to strong full-screen, black screen and hyperlink. However, in order to modify the contents of PPT or to open a file when using a flip laser pointer, a mouse is needed. Therefore, the mouse has also been one of the most widely used human-computer interaction devices. From the early wheeled mouse to the optical mouse, to today's wireless mouse, mouse has become more powerful and more convenient to use. For example, not only does the optical mouse make the cursor move more sensitively and accurately, but also it avoids the abrasion of mechanical wheel during the long-term use. And the wireless mouse breaks through the constraints of wire, which makes human-computer interaction become freer. However, all the three kinds of mice are limited to use on a desk, which makes the human-computer interaction inconvenient on some occasions.

Chen et al. [1] designed a three-dimensional wireless mouse, using the attitude measurement property of 3 axis accelerometer. Li et al. [2] designed a 3D mouse based on three-axis accelerometer ADXL345 and Cortex-M0 processor. However, the experiment results show that the MEMS sensors are easily influenced by noise, as a result of which, it is difficult to collect accurate displacements using a MEMS accelerometer. Tang $e t$ al. [3] measured the inclination angles in static or quasi-static environments by triaxial acceleration sensors, and made the mouse move on a two-dimensional plane by changes of two inclination angles, but only partial noise can be eliminated. In reference [4], a mouse using MEMS inertial sensors was proposed, which used the characteristics of sensor inclination angle and direction angle to control the mouse moving in three-dimensional space, and the test results on Windows platform verified the feasibility of the design. However the mouse does not break through the constraints of the connecting line.

In order to improve the convenience and efficiency of human-computer interaction, a new threedimensional wireless human-computer interaction controller, which effectively combines the wireless air mouse and laser pointer, is designed. Large numbers of experiment results verify the effectiveness of the controller.

\subsection{Hardware system overview}

\section{Hardware System Configuration}

The control system includes a transmitting device and a receiving device, and mainly consists of microcontroller, three-dimensional motion collection module, $2.4 \mathrm{GHz}$ wireless module, mouse driver module, multimedia presentation module and voltage regulator module. The system configuration is shown in Fig.1.

MSP430F149 chip is used as the core of the microcontroller, and the three-dimensional motion collection module based on MPU6050 chip can transmit data to the MSP430F149 controller via serial communication protocol. Wireless transmitting and receiving module are realized based on the $2.4 \mathrm{GHz} \mathrm{RF}$ chip NRF24L01+ and communicates with the controller via SPI protocol. Multimedia presentation module consists of laser light, page up and down keys and so on.

* Correspondence author, mxyuan78@hotmail.com. 


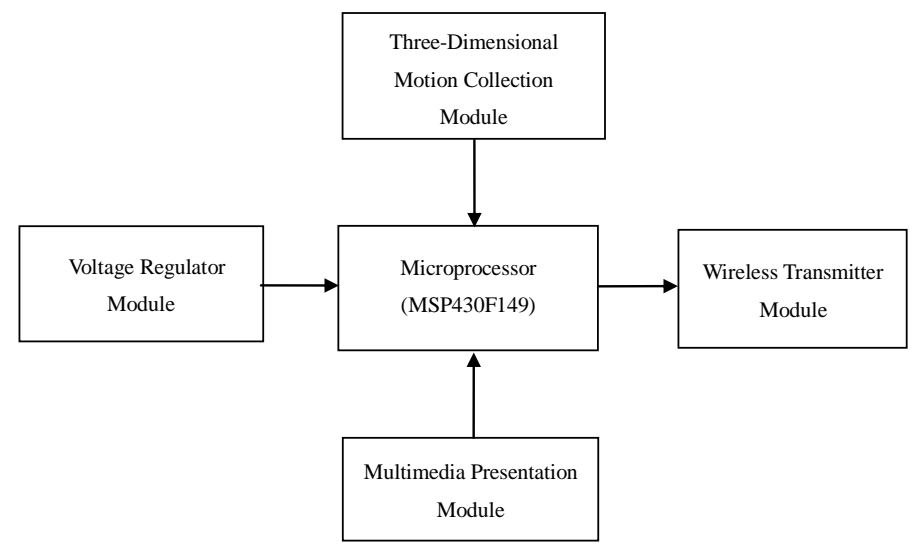

(a)Transmitting device

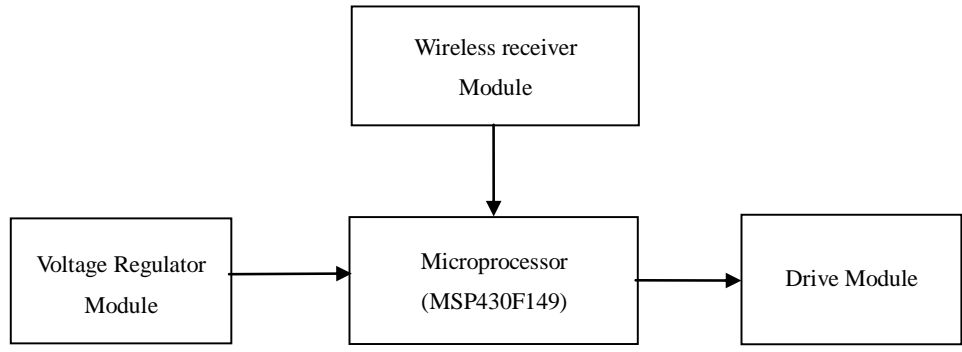

(b) Receiving device

Fig.1 Hardware System Configuration

\subsection{Three-dimensional motion collection module}

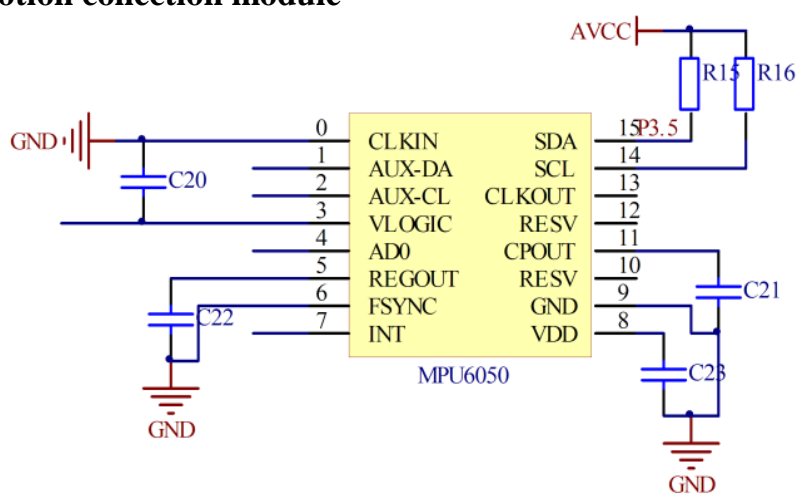

Fig.2 6-axis Inertial Navigation Module

Because the movement control of the cursor is achieved by rotating and tilting movement of the wrist in the human-computer interaction system, a position sensor chip is needed in the system. The 6-axis inertial navigation module based on the gyroscope and accelerometer chip MPU6050 is used in this design, and the module integrating attitude solver can output the data of current attitude by using dynamic Kalman filter algorithm. The peripheral circuit of MPU6050 is shown in Fig.2.

Because MPU6050 module supports the serial communications, its SDA pin is connected with P3.5 pin of MSP430F149. Then the microprocessor can get the corresponding data of acceleration, angular velocity and attitude angle.

\subsection{Wireless transmitting and receiving module}

In order to break through the constraint of wire, the interaction system transmits data by wireless communication. The motion and keys data collected by the microprocessor MSP430 in transmitting device are transmitted wirelessly to the receiving device, thus the corresponding response is realized. In this design, a single chip $2.4 \mathrm{GHz}$ transceiver nRF24L01+ [5] with enhanced SchockBurst mode is chosen. The setting regarding the selection and protocol can be finished through the Serial Peripheral Interface (SPI). The peripheral circuit of wireless transmitting module is shown in Fig.3. 


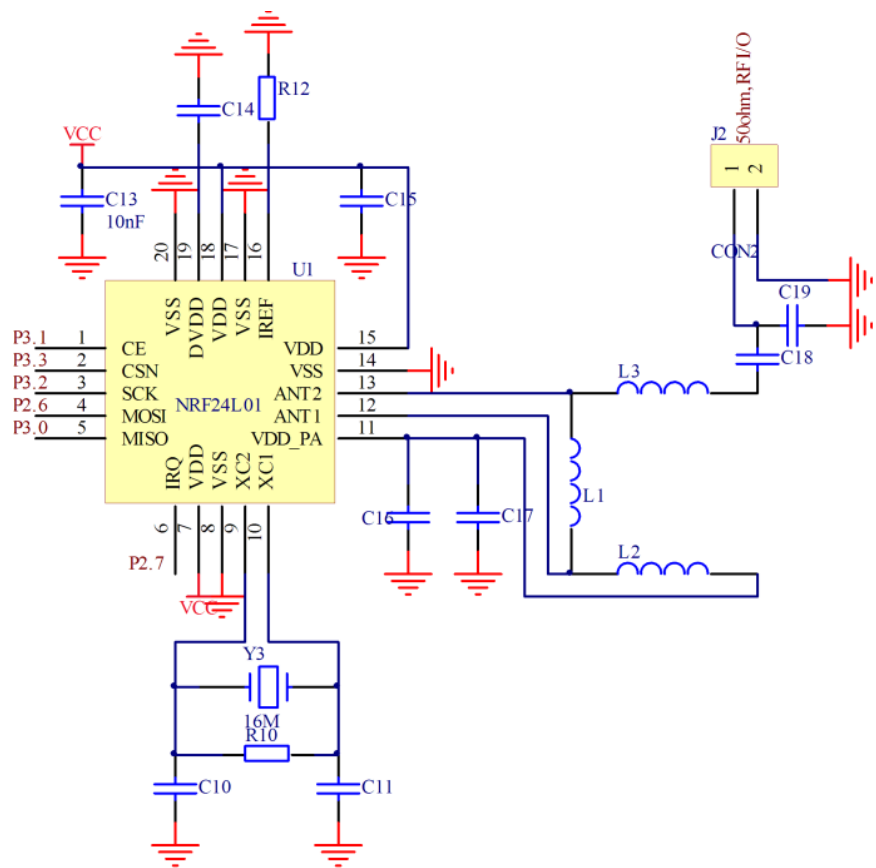

Fig.3 Peripheral circuit of wireless transmitting module

In order to ensure the accuracy of data transmission, the enhanced SchockBurst mode is used in this design. The enhanced ShockBurst mode can control the acknowledgement of received packets and retransmissions of lost packets without increasing workload of MCU.

\subsection{Drive Module}

In this interaction system, the functions of left, right, page up and page down keys are realized based on the driver module. At present, the PL2303 chip is more widely used; however the complex driver needs to be written. In order to improve the development efficiency, HT82M98A chip is chosen in this design, and it just needs to be connected to the PC by USB cable. The external circuit of HT82M98A is as shown in Fig.4.

From the figure, it can be seen that the $\mathrm{X} 1, \mathrm{X} 2, \mathrm{Y} 1, \mathrm{Y} 2, \mathrm{Z} 1, \mathrm{Z} 2, \mathrm{R}, \mathrm{L}$ pins of driver module are connected to the P2 and P6 ports of MSP430 respectively. The cursor and the wheel can be controlled by controller through the $\mathrm{X} 1, \mathrm{X} 2, \mathrm{Y} 1, \mathrm{Y} 2$ pins.

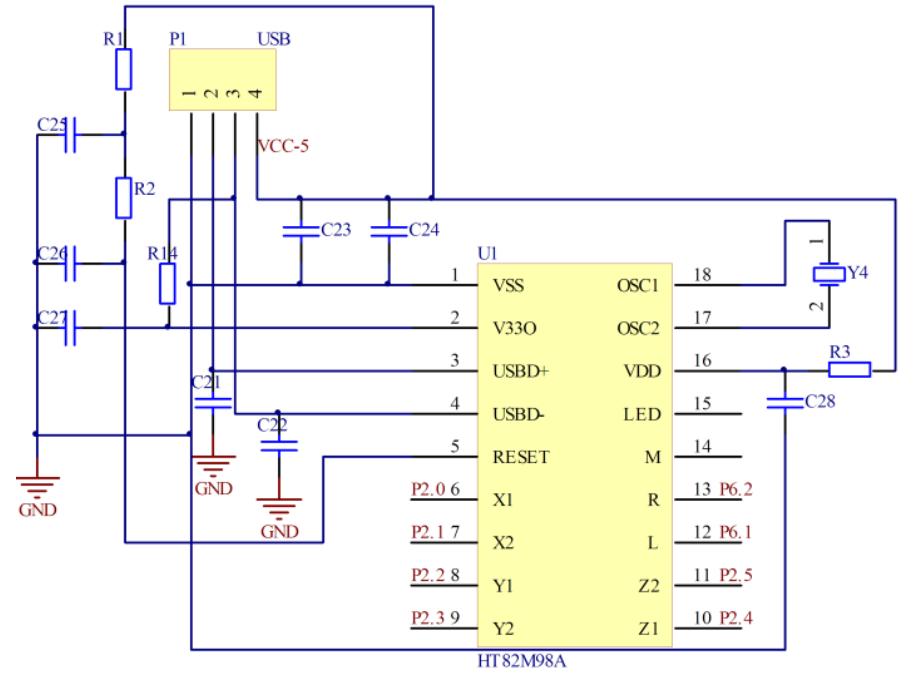

\subsection{Schematic}

Fig.4 External circuit of HT82M98A driver module

As described above, the human-computer interaction system includes a transmitting device and a receiving device. Fig.5(a) shows the transmitting circuit, and Fig.5(b) shows the receiving circuit. 


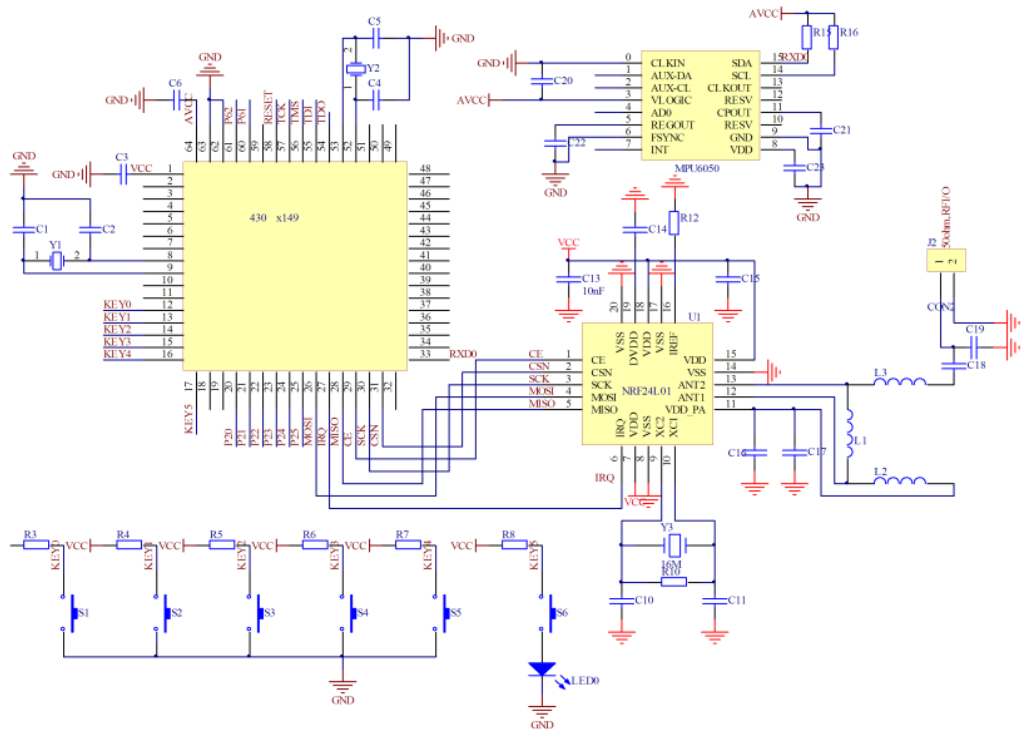

(a)Transmitter circuit

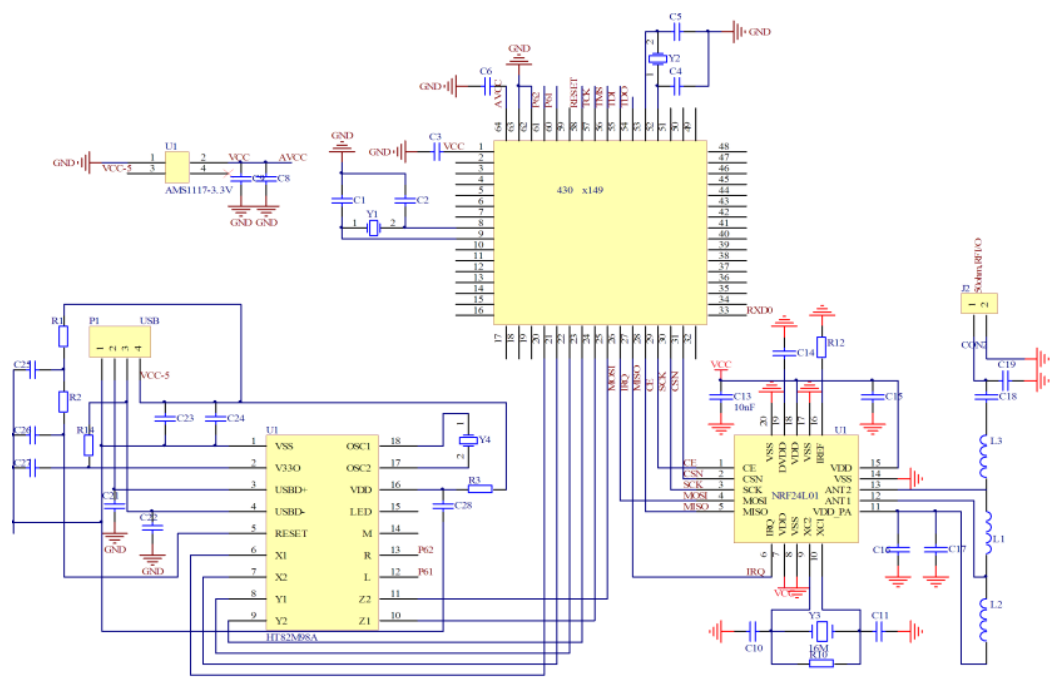

(b)Receiver circuit

Fig.5 Schematic

\subsection{Circuit Board}

Fig. 6(a) shows the final transmitting circuit board made according to transmitter schematic, and Fig. 6(b) shows the final receiving circuit board made according to the receiving schematic.

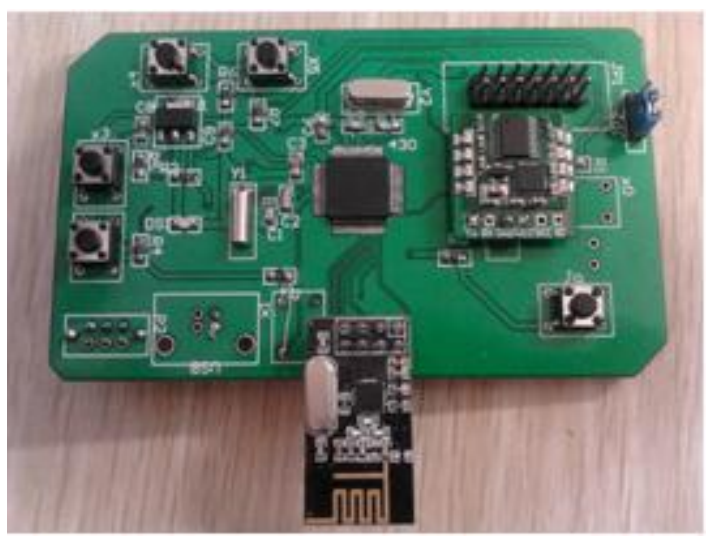

(a)Transmitting circuit board

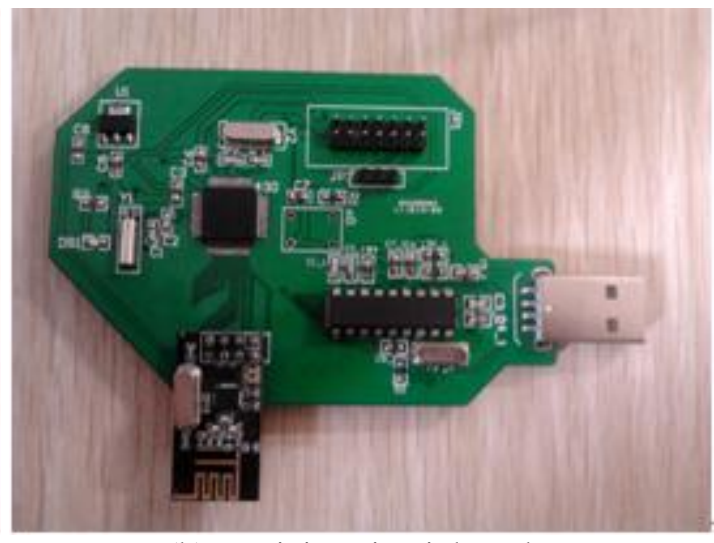

(b)Receiving circuit board

Fig. 6 Circuit board 


\section{Experimental Test And Analysis}

In order to verify the effectiveness of human-computer interaction system, two experiments regarding three-dimensional air mouse and multimedia page are executed respectively. Figs.7(a)-(f) show the drawing process of block diagram by the left key operation and wrist movement.

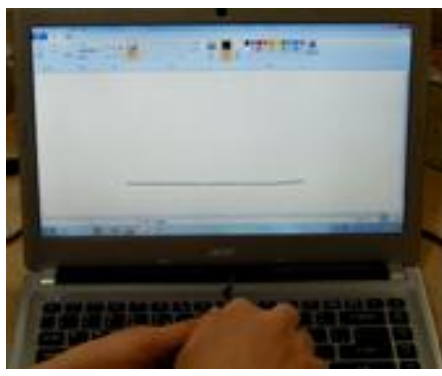

(a)

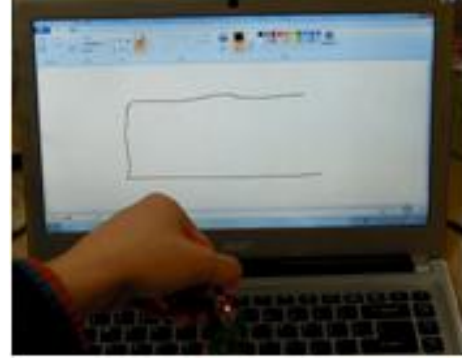

(d)

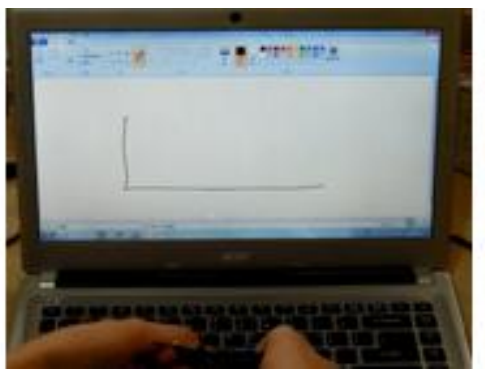

(b)

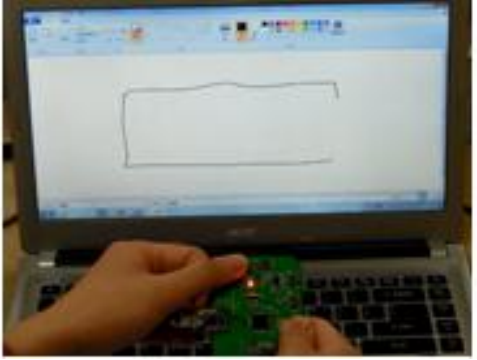

(e)

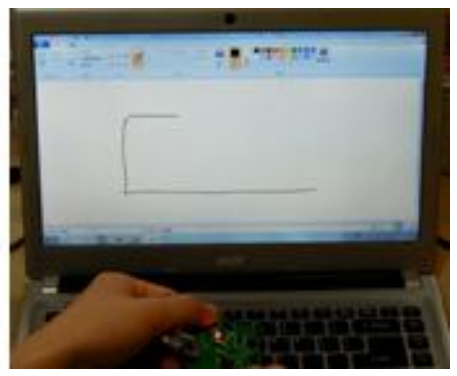

(c)

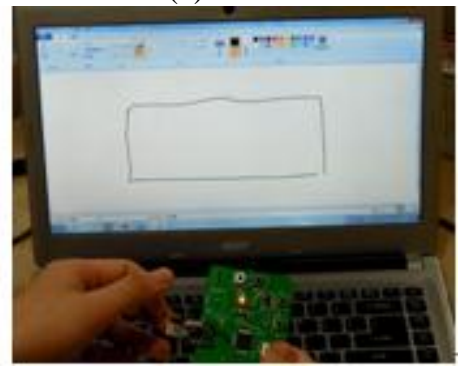

(f)

Fig.7 Experiment of three-dimensional air mouse

Figs.8 (a)-(f) show the function of multimedia player by using the page up and page down keys.

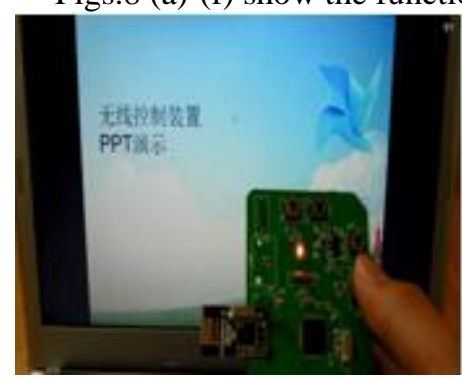

(a)

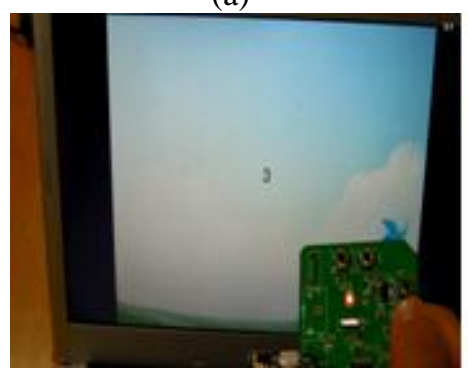

(d)

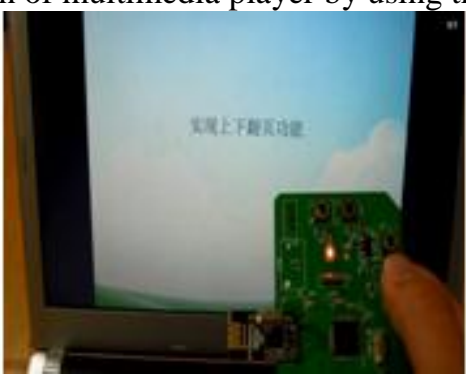

(b)

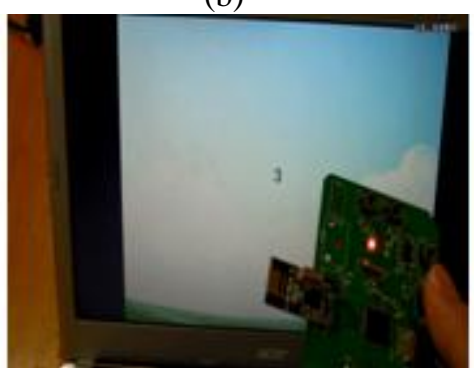

(e)

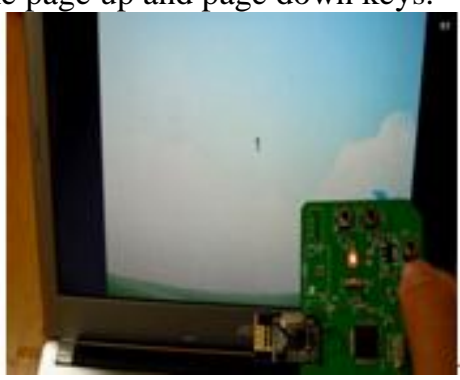

(c)

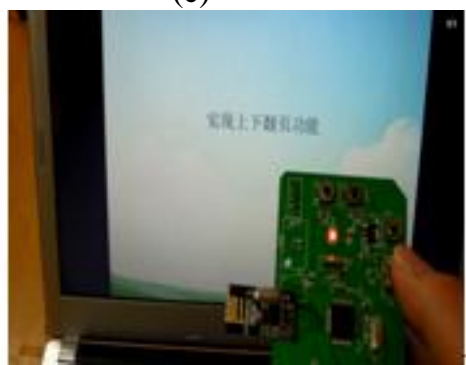

(f)

Fig.8 Experiment of multimedia page

From the figures, it can be seen that the interaction system can be used flexibly at three-dimensional space through wireless communication.

\section{Conclusion}

For the requirements of the convenience and efficiency of human-computer interaction, a new humancomputer interaction control system is designed and developed in this paper. The hardware system configuration of the device, which consists of system components, peripheral circuits of each module, the system schematic and final circuit board, is introduced firstly. Then the experiments of three-dimensional operation and multimedia page are showed and verify the validity of the interaction device. The device combines the functions 
of three-dimensional wireless mouse and laser pointer and meets the needs of human-computer interaction during the multimedia teaching and conference.

\section{References}

[1] J.X. Chen, X. Bu, R. Wang, J. Liu and Y. Zhang, The design and implementation of MEMS accelerometer-based 3D wireless mouse, Wireless Internet Technology, 8, 2011, 22-25.

[2] B.C. Li, W.X. Ning, Exploration and design of 3-axis accelerometer-based 3D mouse, Computer CD Software and Applications, 9, 2013, 122-123.

[3] J. Tang, W.Y. Chen, X.B. Jiang, L. Qiang, Design of micro-accelerometer mouse system, Computer Engineering and Design, 29(23), 2008, 6136-6138.

[4] J.X. Chen, J. Zang, L.L. Yang, Design and implementation of three dimension mouse based on MEMS sensor, Computer Engineering, 38(24), 2012, 274-278

[5] B. Guo, S.F. Luo, X.D. Li, Design of TV remote controller with mouse function based on NRF24L, Electronic Engineering \& Product World, 6, 2011, 49-51.

[6] C.H. Zhou, X.K. Li, D.W. Han, Wireless mouse circuit design and implementation of algorithm, Computer Measurement \& Control, 20(12), 2012, 3329-3331. 